\title{
Cardioprotective effects of the serine protease inhibitor aprotinin after regional ischemia and reperfusion on the beating heart
}

\author{
Diethard Pruefer, $\mathrm{MD}^{\mathrm{a} *}$ \\ Ute Buerke, $M D^{\text {b* }}$ \\ Markus Khalil, MD \\ Manfred Dahm, MD \\ Harald Darius, $M D^{\mathrm{b}}$ \\ Hellmut Oelert, $\mathrm{MD}^{\mathrm{a}}$ \\ Michael Buerke, MD ${ }^{b}$
}

\footnotetext{
From the Department of Cardiothoracic and Vascular Surgery ${ }^{\mathrm{a}}$ and II Department of Medicine-Cardiology, ${ }^{\mathrm{b}}$ Johannes Gutenberg-University, Mainz, Germany.

Supported in part by grant Bu 819/3-1 of Deutsche Forschungsgemeinschaft and Robert Mueller-Foundation, Germany (U.B., M.K., H.D., and M.B.).

Received for publication Oct 18, 2001; revisions requested Dec 10, 2001; revisions received Jan 15, 2002; accepted for publication Feb 10, 2002.

Address for reprints: Michael Buerke, MD, II Department of Medicine-Cardiology, Johannes Gutenberg-University, LangenbeckStr 1, D-55101 Mainz, Germany (E-mail: buerke@mail.uni-mainz.de).

*These authors made equal contributions.

J Thorac Cardiovasc Surg 2002;124:942-9

Copyright () 2002 by The American Association for Thoracic Surgery

$0022-5223 / 2002 \$ 35.00+0 \quad \mathbf{1 2 / 1 / 1 2 3 7 0 3}$

doi: $10.1067 / \mathrm{mtc} .2002 .123703$
}

Objective: Early coronary reperfusion of the ischemic myocardium is a desired therapeutic goal to preserve myocardium. However, reperfusion itself contributes to an additional myocardial injury (ie, reperfusion injury), which has been attributed to neutrophil infiltration with subsequent release of proteases and oxygen-derived radicals. We studied the effects of the serine protease inhibitor aprotinin (Trasylol) on myocardial ischemia and reperfusion in a rat model.

Methods: The effects of aprotinin (5000 and 20,000 U/kg) were examined in vivo in a rat model of regional myocardial ischemia (20 minutes) and long-term reperfusion ( 24 hours). Cardioprotecive effects were determined by means of measurement of creatine kinase and myeloperoxidase activity within the myocardium, as well as histochemical analysis.

Results: Aprotinin (20,000 U/kg) administrated 2 minutes before reperfusion significantly attenuated myocardial injury expressed as creatine kinase washout compared with that seen in vehicle-treated rats $(65 \pm 25$ vs $585 \pm 98$ creatine kinase difference in units per $100 \mathrm{mg}, P<.01$ ). Administration of $5000 \mathrm{U} / \mathrm{kg}$ of the protease inhibitor resulted in partial inhibition of myocardial reperfusion injury. Moreover, cardiac myeloperoxidase activity in the ischemic myocardium, a marker of neutrophil accumulation, was significantly reduced after aprotinin treatment. Histologic analysis of the reperfused myocardium demonstrated reduced polymorphonuclear leukocyte infiltration and reduced tissue injury. Furthermore, aprotinin treatment resulted in decreased induction of cardiac myocyte apoptosis compared with that seen in vehicle-treated rats.

Conclusions: Inhibition of serine proteases with aprotinin appears to be an effective means of preserving ischemic myocardium from reperfusion injury, even after 24 hours of reperfusion. Aprotinin might exert cardioprotection through inhibition of polymorphonuclear leukocyte-induced myocardial injury and inhibition of reperfusion-induced apoptosis of cardiac myocytes.

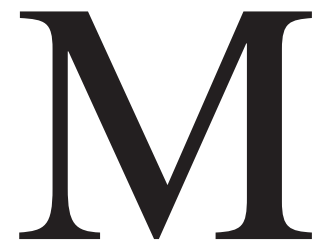

yocardial ischemia (MI) without reperfusion results in severe cardiac dysfunction and myocyte necrosis. Therefore, early reperfusion of the ischemic myocardium is the desired goal. Evidence indicates that reperfusion itself contributes to an additional myocardial injury in ischemic hearts (ie, reperfusion injury). ${ }^{1}$ This injury is preceded by loss of endothelial function and accumulation of activated neutrophils into the ischemic-reperfused 
myocardium (ie, inflammatory injury). The inflammatory component to ischemia-reperfusion injury can trigger both necrosis and apoptosis, in which necrosis itself is an inflammatory-inducing process, compared with apoptosis, which does not result in an inflammatory response. Several studies have shown that neutrophils adhere to the vascular endothelium, extravasate, and accumulate into ischemic-reperfused tissue., ${ }^{2,3}$ These activated neutrophils cause tissue injury by releasing cytotoxic metabolites, including proteases (ie, cathepsin G and elastase), oxygen-derived free radicals (ie, superoxide and hydrogen peroxide), eicosanoids, and cytokines. ${ }^{4}$ Several therapeutic strategies have been studied in regard to limiting the inflammatory response after MI/R, and recent attention has been focused on serine protease inhibitors.

Serine proteases, such as elastase-related proteinases (ie, cathepsin), use serine side chain hydroxyl groups to attack and cleave peptide bonds in proteins. ${ }^{5}$ Cathepsin $\mathrm{G}$ and the elastase-related proteases are the major neutral serine proteases present in azurophil granules of neutrophils and monocytes. ${ }^{4}$ However, the exact mechanism and biologic relevance of many proteases is not precisely understood. Exogenous serine protease inhibitors have been studied in an attempt to limit polymorphonuclear neutrophil (PMN)mediated injury. Mulligan and colleagues ${ }^{6}$ demonstrated $^{2}$ that inhibition of elastase and cathepsin $G$ significantly reduces PMN-mediated lung injury.

The serine protease inhibitor aprotinin (Trasylol) belongs to a group labeled as Kunitz protease inhibitors because of its common Kunitz domain in its polypeptide structure. Aprotinin has a molecular weight of $6512 \mathrm{~d}$ and is a naturally occurring broad-spectrum serine proteinase inhibitor obtained from bovine lungs. It consists of 58 amino acid residues that are arranged in a single polypeptide chain cross-linked by 3 disulfides. Aprotinin has also been reported to inhibit neutrophil elastase release ${ }^{7}$ and superoxide anion formation, ${ }^{8}$ suggesting an effect on neutrophil activation. Previous studies demonstrated cardioprotective effects in acute MI without reperfusion ${ }^{9}$ or short-term reperfusion. ${ }^{10}$ Other in vitro studies have demonstrated the important role of PMN elastase in reperfusion of the ischemic bowel, ${ }^{11}$ in elastase-mediated damage of cultured vascular endothelial cells, ${ }^{12}$ and in isolated cardiac myocytes. ${ }^{13}$ However, the role of myocardial serine protease inhibitors in myocardial ischemia and reperfusion (MI/R) and potential cardioprotective effects are still under investigation.

Therefore the primary objectives of the present study were to determine (1) the in vivo effects of the exogenous serine protease inhibitor aprotinin on myocardial tissue necrosis, (2) neutrophil accumulation in the ischemic-reperfused myocardium, and (3) induction of postreperfusion apoptosis in a well-established model of rat MI and longterm reperfusion.

\section{Materials and Methods}

\section{Experimental Protocol}

Male Sprague-Dawley rats (225-250 g) were anesthetized with ether before the operation. A skin incision was made over the left thorax, and pectoral muscles were retracted to expose the ribs. A purse-string suture was placed loosely through the skin and underlying musculature to facilitate rapid closure of the chest wall. A thoracotomy was performed at the level of the fifth intercostal space. MI was produced by briefly exteriorizing the heart and placing a 4-0 silk suture around the left coronary artery, approximately 2 to $3 \mathrm{~mm}$ from its origin, effectively occluding the vessel. Because the rat has low coronary collateral circulation, this method results in a uniform degree of myocardial injury. After the slipknot was tied, the heart was replaced in the thoracic cavity; air was evacuated from the thorax; the chest wall, muscles, and skin were rapidly closed by using the previously placed purse-string sutures; and the rats were allowed to breath spontaneously. These procedures are routinely completed in less than 60 seconds. Left ventricular free wall (LVFW) is subjected to severe ischemia. After 20 minutes of MI, the slipknot was released, initiating reperfusion of the ischemic myocardium. Sham-operated control rats (sham MI) underwent the same surgical procedures, except that the suture, which was passed under the left coronary artery, was not tied. After the surgical procedure, the rats were observed for 2 hours and than placed in a cage for the remaining 24 hours (eg, reperfusion time).

Rats were randomly divided into 4 major groups, consisting of the following: (1) sham MI rats receiving aprotinin (Trasylol; Bayer, Leverkusen, Germany; 20,000 U/kg body weight; $\mathrm{n}=9$ ); (2) $\mathrm{MI} / \mathrm{R}$ rats receiving vehicle $(\mathrm{NaCl} 0.9 \%, \mathrm{n}=8)$; (3) $\mathrm{MI} / \mathrm{R}$ rats receiving $5000 \mathrm{U} / \mathrm{kg}$ aprotinin $(\mathrm{n}=10)$; and (4) MI/R rats receiving $20,000 \mathrm{U} / \mathrm{kg}$ aprotinin $(\mathrm{n}=9)$. Aprotinin or its vehicle were administered by means of single intravenous bolus injection 2 minutes before reperfusion of the ischemic myocardium.

After 24 hours of reperfusion, rats were anesthetized with pentobarbital sodium, and their hearts were excised and placed in oxygenated Krebs-Henseleit (K-H) solution at $4^{\circ} \mathrm{C}$ and $\mathrm{pH}$ 7.4. The K-H solution consisted of the following: $\mathrm{NaCl}, 118 \mathrm{mmol} / \mathrm{L}$; $\mathrm{KCl}, 4.75 \mathrm{mmol} / \mathrm{L} ; \mathrm{CaCl}_{2} \cdot 2 \mathrm{H}_{2} \mathrm{O}, 2.54 \mathrm{mmol} / \mathrm{L} ; \mathrm{KH}_{2} \mathrm{PO}_{4}, 1.19$ $\mathrm{mmol} / \mathrm{L} ; \mathrm{NaHCO}_{3}, 12.5 \mathrm{mmol} / \mathrm{L}$; and glucose, $10.0 \mathrm{mmol} / \mathrm{L}$. Rats showing major bleeding inside the thoracic cavity or ineffective reperfusion (ie, persisting ligature) were excluded from the study. All hearts were examined at the site of the ligature to obtain proper reperfusion after regional ischemia. In detail, the hearts were investigated with a stereomicroscope to determine whether the ligature was loose and whether there was proper reperfusion. After 24 hours, the exclusion rate and mortality rate together were less than $15 \%$. The aorta, atrium, right ventricle, and adherent fat or connective tissue were dissected from the left ventricle. The LVFW (ischemic-reperfused myocardium) and interventricular septum (IVS; nonischemic control myocardium) were separated and homogenized in cold $0.25 \mathrm{~mol} / \mathrm{L}$ sucrose containing $1 \mathrm{mmol} / \mathrm{L}$ ethylenediamine tetraacetic acid (EDTA) and $1 \mathrm{mmol} / \mathrm{L}$ mercaptoethanol with an Ultra-Turrax tissue homogenizer (Jahnke + Kunkel, Staufen, Germany). Homogenates were centrifuged at $36,000 \mathrm{~g}$ at $4^{\circ} \mathrm{C}$ for 30 minutes. The supernatants were decanted and analyzed spectrophotometrically for myocardial creatine kinase (CK) content and myeloperoxidase (MPO) activities. 
In additional sham rats $(\mathrm{n}=5)$ we determined the hemodynamic parameters and white blood cell counts after aprotinin administration. There were no significant changes in any of the cardiovascular variables (heart rate and blood pressure) or any significant changes in white blood cell counts measured in shamoperated rats treated with $20,000 \mathrm{U} / \mathrm{kg}$ aprotinin bolus injection.

All experiments were approved by the University Animal Care Committee.

\section{Myocardial CK Analysis}

The CK washout (or CK loss) from the injured left ventricle has been shown to be a useful marker for myocyte injury after MI/ R. ${ }^{14,15}$ Lefer and colleagues ${ }^{15}$ showed that peak myocardial CK loss occurred 24 hours after myocardial reperfusion. Thus we compared myocardial CK activity between the ischemic-reperfused LVFW and the control nonischemic IVS. CK activity of LVFW and septum was measured by using the method of Rosalki. ${ }^{16}$ The supernatants of the homogenized myocardium (ie, LVFW and septum) were incubated with adenosine diphosphate and phosphocreatine (Sigma Chemical Co, Deisenhofen, Germany), and the change in absorbance was measured spectrophotometrically at $340 \mathrm{~nm}$. Myocardial CK loss after MI/R was calculated in each rat by subtracting $\mathrm{CK}_{\mathrm{LVFW}}$ from $\mathrm{CK}_{\mathrm{IVS}}$ and expressed in international units per milligram of protein. The protein concentration was determined by using the biuret method of Gornall and colleagues. ${ }^{17}$

In additional rats $(n=5)$ receiving vehicle, the above-described procedures were performed, except that one half of the LVFW was incubated with $20,000 \mathrm{U} / \mathrm{kg}$ aprotinin or an equal volume of $0.9 \% \mathrm{NaCl}$ to determine whether aprotinin altered the CK assay. These values were not significantly different, indicating that aprotinin had no direct effect on the CK assay. All assays were measured without prior knowledge of the group of origin of each rat.

\section{Determination of Myocardial MPO Activity}

The myocardial activity of MPO, an enzyme occurring virtually exclusively in neutrophils, was determined by using the method of Bradley and colleagues, ${ }^{18}$ as modified by Mullane and associates. ${ }^{19}$ Change in absorbance was measured spectrophotometrically in the supernatants of homogenized myocardium (ie, LVFW and IVS). One unit of MPO is defined as the quantity of enzyme hydrolyzing $1 \mathrm{mmol}$ of peroxide per minute at $25^{\circ} \mathrm{C}$. The elevation of the MPO activity in the LVFW was calculated by subtracting $\mathrm{MPO}_{\text {IVS }}$ from $\mathrm{MPO}_{\mathrm{LVFW}}$ and expressed as cardiac MPO difference (in units per $100 \mathrm{mg}$ tissue weight.

In additional rats $(n=4)$ receiving vehicle, the above-described procedures were performed, except that one half of the necrotic tissue was incubated with $20,000 \mathrm{U} / \mathrm{kg}$ aprotinin or an equal volume of $0.9 \% \mathrm{NaCl}$ to determine whether aprotinin altered the MPO assay. These values were not significantly different, indicating that aprotinin had no direct effect on the MPO assay. All MPO determinations were made without prior knowledge concerning the group of treatment of each rat.

\section{Histologic Analysis of Neutrophil Infiltration and Myocardial Tissue Injury}

Rat hearts were removed at the end of the reperfusion period and immediately cannulated through the aorta. The hearts were per- fused for 2 minutes with K-H buffer (ie, until the heart was cleared of blood). After the hearts were cleared of blood, perfusion was switched to $4 \%$ paraformaldehyde in phosphate-buffered saline ( $\mathrm{pH} 7.4$ at $4^{\circ} \mathrm{C}$ ) for 5 minutes to perfusion fix the hearts. Fullthickness slices of the myocardial tissue were fixed for 1.5 hours at $4{ }^{\circ} \mathrm{C}$ in $4 \%$ paraformaldehyde. After 1.5 hours, the slices were dehydrated in a graded series of acetone (ie, 50\%, 70\%, 90\%, and $100 \%$ ) at $4{ }^{\circ} \mathrm{C}$. After dehydration, the sections were infiltrated with methacrylate (Immunobed; Polyscience, Eppelheim, Germany) at room temperature for 24 hours and subsequently embedded in methacrylate at $4^{\circ} \mathrm{C}$ for 12 hours. Glass knives were used to cut 5 - $\mu \mathrm{m}$-thick tissue sections. The tissue sections were placed on Vectabond-coated slides (Vector Laboratories, Burlingame, Calif). The sections were stained with Gill's hematoxylin solution (Sigma Co) and examined with a Zeiss microscope (Zeiss, Göttingen, Germany). The numbers of PMNs in 5 fields of each slide were counted from each heart. Three rats were studied in each group.

\section{In Situ Determination of Apoptosis in Ischemic Reperfused Myocardium}

Because reperfusion of ischemic myocardium results in necrotic tissue injury, as well as cardiac myocyte apoptosis, we performed in situ detection of myocardial apoptosis. Rats were subjected to sham ischemia or 20 minutes of ischemia followed by 24 hours of reperfusion and given either 20,000 $\mathrm{U} / \mathrm{kg}$ aprotinin $(\mathrm{n}=5)$ or its vehicle $(n=5)$. At the end of reperfusion period, the hearts were removed and perfused retrogradely with K-H buffer for 2 minutes. Myocardium was perfused, fixed, embedded, and cut, as described previously.

The presence of apoptosis in the ischemic-reperfused myocardium was determined by means of direct immunoperoxidase detection of digoxigenin-labeled genomic DNA in thin sections of myocardium (ApopTag; Oncor, Gaithersburg, Md). Tissue sections were treated with trypsin and $\mathrm{H}_{2} \mathrm{O}_{2}$. Residues of digoxigenin nucleotide were catalytically ( 1 hour at $37^{\circ} \mathrm{C}$ ) added to the $3^{\prime}-\mathrm{OH}$ end of DNA by means of terminal deoxynucleotidyltransferase. Incubation with the anti-digoxigenin antibody fragment was carried out for 30 minutes at room temperature. Color development was performed with a diaminobenzidine substrate $/ \mathrm{H}_{2} \mathrm{O}_{2}$ solution (Vector Laboratories). The sections were lightly counterstained with hematoxylin and examined with a Zeiss light microscope (Zeiss). Because sometimes necrotic myocytes express positive TUNEL staining, we analyzed only myocytes that did not have the typical signs of necrosis (ie, loss of cross-striations and loss of membrane integrity). The percentage of immunolabeled nuclei of myocytes (ie, apoptotic cells) was counted in random tissue sections as an index of occurrence of apoptosis. Myocytes were analyzed in at least 10 separate fields for each tissue section. The number of apoptotic myocytes (ie, peroxidase positive in the cell nucleus) was counted for each field. The number of stained myocytes was divided by the total number of myocytes and then multiplied by 100 to determine the percentage of stained myocytes as follows:

$($ Stained myocytes $) /($ Total myocytes $) \times 100$. 


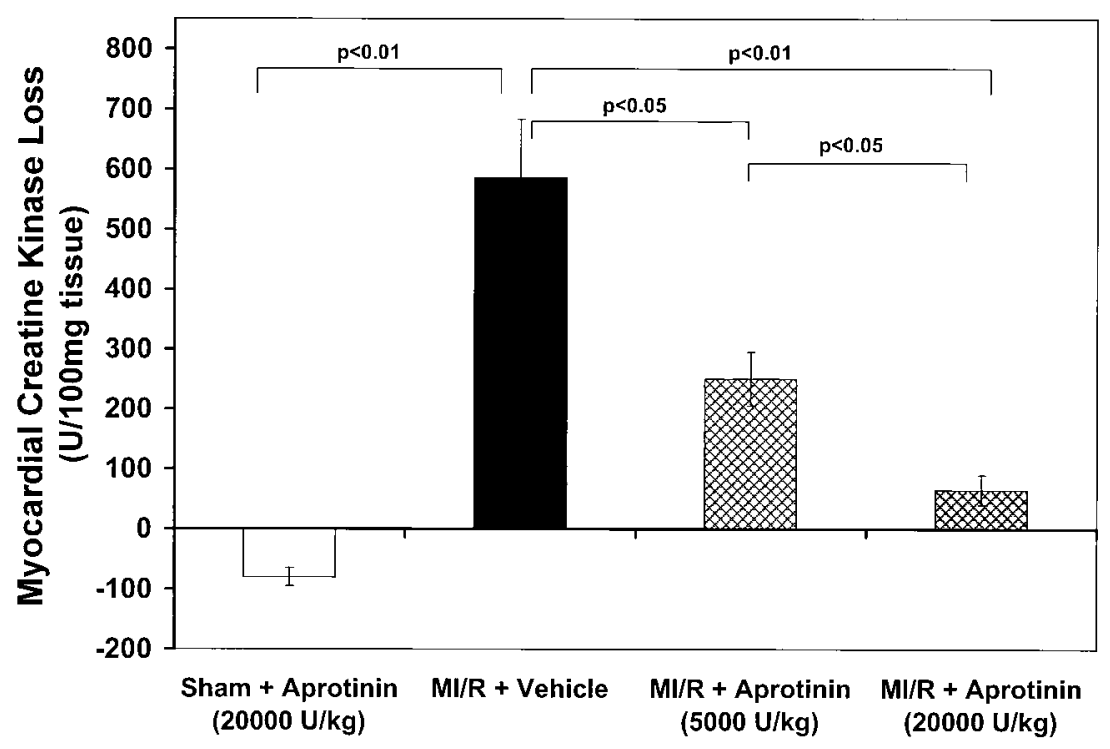

Figure 1. Effect of single administration of aprotinin on myocardial injury after reperfusion of ischemic myocardium. CK activity is calculated as the difference between IVS and LVFW expressed in international units per $100 \mathrm{mg}$. All values are means \pm SEM.

\section{Statistical Analysis}

All values in Figures and text are presented as means \pm SEM (ie, the mean of $n$ independent experiments). All data were subjected to analysis of variance, followed by the Fisher $t$ test.

\section{Results}

\section{Effect of Aprotinin on Myocardial Injury After}

\section{Ischemia and Reperfusion}

To evaluate the extent of myocardial ischemic injury, we measured CK activity in homogenates of nonischemic (ie, IVS) or ischemic (ie, LVFW) myocardial tissue after 24 hours of reperfusion. Sham MI/R rats receiving either vehicle or 20,000 U/kg aprotinin demonstrated no significant difference in CK activity between IVS and LVFW, indicating that no myocyte necrosis occurred as a result of surgical procedures (data not shown). However, rat hearts subjected to coronary occlusion that were given only the vehicle demonstrated significant loss of CK from the LVFW after 24 hours of reperfusion $(P<.01$ compared with sham MI rats, Figure 1). In contrast, bolus injection of aprotinin (either $5000 \mathrm{U} / \mathrm{kg}$ or $20,000 \mathrm{U} / \mathrm{kg}$ ) 2 minutes before reperfusion significantly attenuated dose-dependent CK loss from the ischemic-reperfused LVFW. These results clearly indicate that administration of aprotinin prevents myocardial tissue injury after reperfusion of the ischemic myocardium in vivo.

\section{Neutrophil Accumulation Into the Ischemic}

\section{Reperfused Myocardium}

We measured MPO activity in the nonischemic septum (IVS) and ischemic (LVFW) regions of the myocardium and calculated the difference (LVFW - IVS) between the 2 MPO values as an index of neutrophil accumulation. Shamoperated control rats receiving 20,000 U/kg aprotinin (Figure 2) showed low and similar levels and differences of MPO to those of sham MI/R rats given vehicle or aprotinin (5000 U/kg; data not shown). In contrast, MI rats receiving only the vehicle exhibited a marked increase in MPO activity in the ischemic-reperfused LVFW. These data indicate increased neutrophil accumulation into the reperfused region of the myocardium. However, administration of 5000 and 20,000 U/kg aprotinin intravenously 2 minutes before reperfusion significantly attenuated dose-dependent MPO activity in the ischemic-reperfused myocardium. Similarly, histologic analysis (Figure 3, $A$ and $B$ ) of the ischemicreperfused myocardium confirmed the results of the biochemical assay. These results indicate that the cardioprotective effects of aprotinin might be partially attributed to inhibition of neutrophil accumulation into the ischemicreperfused myocardium.

Immunohistochemical Localization of Apoptosis After Myocardial Ischemia and Reperfusion

The presence of apoptosis in the ischemic-reperfused myocardium was determined by means of direct immunoperoxidase detection of digoxigenin-labeled genomic DNA in thin sections of myocardium. Nonischemic sections of heart tissue or any section from sham MI hearts failed to demonstrate any significant immunostaining. (ie, $<3 \%$ of the myocytes were stained; Figure 4). Similarly, immunohistologic preparations, in which either the digoxigenin-dUTP 


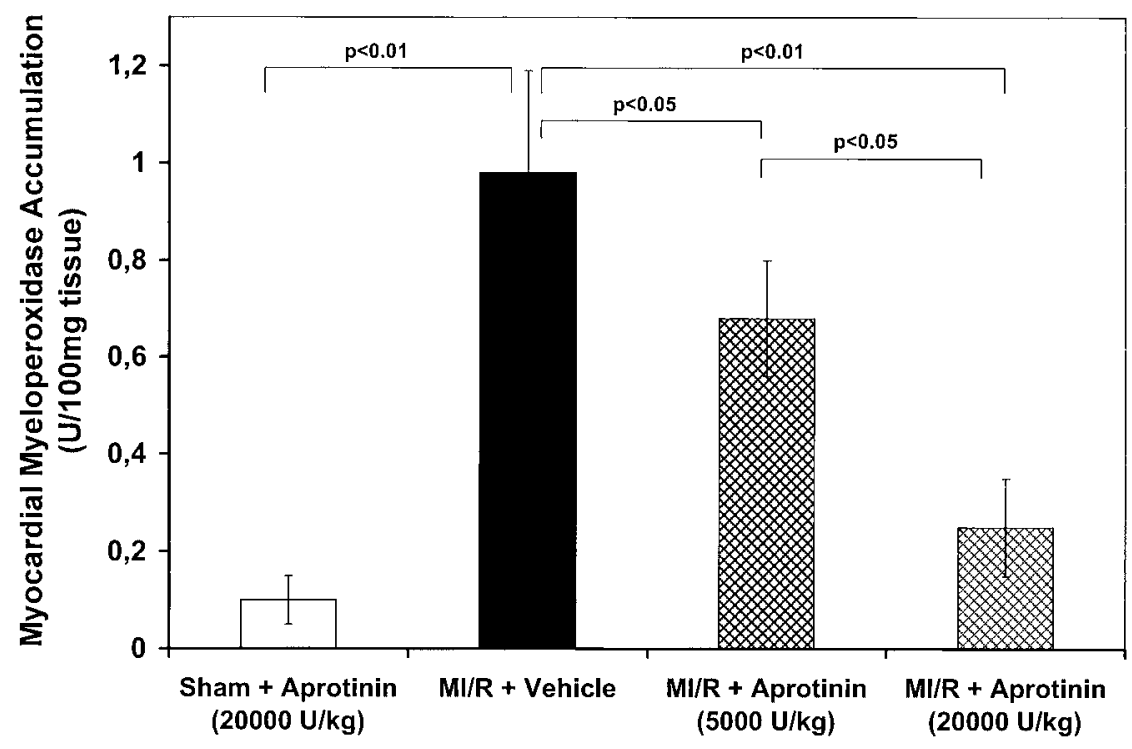

Figure 2. Effect of single administration of aprotinin on neutrophil accumulation after reperfusion of ischemic myocardium. Cardiac MPO as a marker for leukocyte infiltration is expressed as the difference between LVFW and IVS activity. All values are means \pm SEM.

(deoxy-uridinetriphosphate) or the antibody peroxidase conjugate was replaced with nonimmune serum, failed to exhibit any labeling of myocardial or other cell types. In contrast to these controls, apoptosis was clearly evident in ischemic myocardial sections obtained from untreated ischemic-reperfused hearts (ie, $29 \% \pm 4 \%$ positive stained myoctyes; $P<.01$ compared with sham MI rats). Brown reaction product was present in nuclei of apoptotic cells. Apoptosis occurred in single myocytes, and there was no close relation to infiltrating neutrophils in close proximity to the coronary vasculature detectable. Intense immunostaining was evident in myocytes, as well as in infiltrating leukocytes. Ischemic-reperfused myocardium of rats treated with $20,000 \mathrm{U} / \mathrm{kg}$ aprotinin demonstrated a marked reduction of immunostaining (ie, $15 \% \pm 3 \% ; P<.05$ compared with rats receiving only vehicle), indicating reduced occurrence of apoptosis after aprotinin treatment (Figure 3, $C$ and $D)$. In myocardium of vehicle-treated animals, we also observed infiltrating leukocytes undergoing apoptosis. Because aprotinin treatment reduced leukocyte accumulation, the number of apoptotic leukocytes was lower. These results demonstrate that reperfusion of ischemic myocardium induces cardiac myocyte apoptosis and that inhibition of serine proteases with aprotinin appears to be an effective inhibitor of cardiac myocyte apoptosis induction.

\section{Discussion}

Our results clearly demonstrate significant cardioprotective activities of the serine protease inhibitor aprotinin after reperfusion of ischemic myocardium. Aprotinin demon- strated cardioprotective effects in PMN-mediated tissue damage and cardiac myocyte apoptosis in a model of cardiac ischemia and long-term reperfusion in vivo. The cardioprotection exerted by aprotinin was characterized by profound reduction of myocardial necrosis, significant inhibition of PMN accumulation into the reperfused myocardium, and diminished induction of cardiac myocyte apoptosis compared with that seen in rats given only the vehicle. The cardioprotective effects can be partially attributed to inhibition of complement activation and beneficial effects on the protease-mediated cascade initiated by PMN activation, resulting in inhibition of leukocyte accumulation.

PMNs play a major role in endothelial injury of coronary vessels, as well as in the propagation of myocardial necrosis. This is consistent with previous experimental findings obtained in other species. ${ }^{20,21}$ Neutrophils might plug capillaries and thus contribute to the no-reflow phenomenon after reperfusion, thus exacerbating reperfusion-induced myocardial necrosis. Neutrophils that adhere to the vascular endothelium are subsequently activated and release cytotoxic metabolites, such as oxygen free radicals, proteolytic enzymes, and cytokines. ${ }^{4}$ These mediators might further lead to coronary endothelial injury, disruption of the subendothelial basement membrane, and subsequent PMN extravasation and myocardial necrosis., ${ }^{2,3}$

The reduction in tissue injury (ie, loss of CK) exerted by aprotinin cannot be attributed to hemodynamic effects because bolus injection did not alter hemodynamic parameters. Variations in collateral flow in the ischemic myocardium as the cause of the cardioprotective effects are highly 

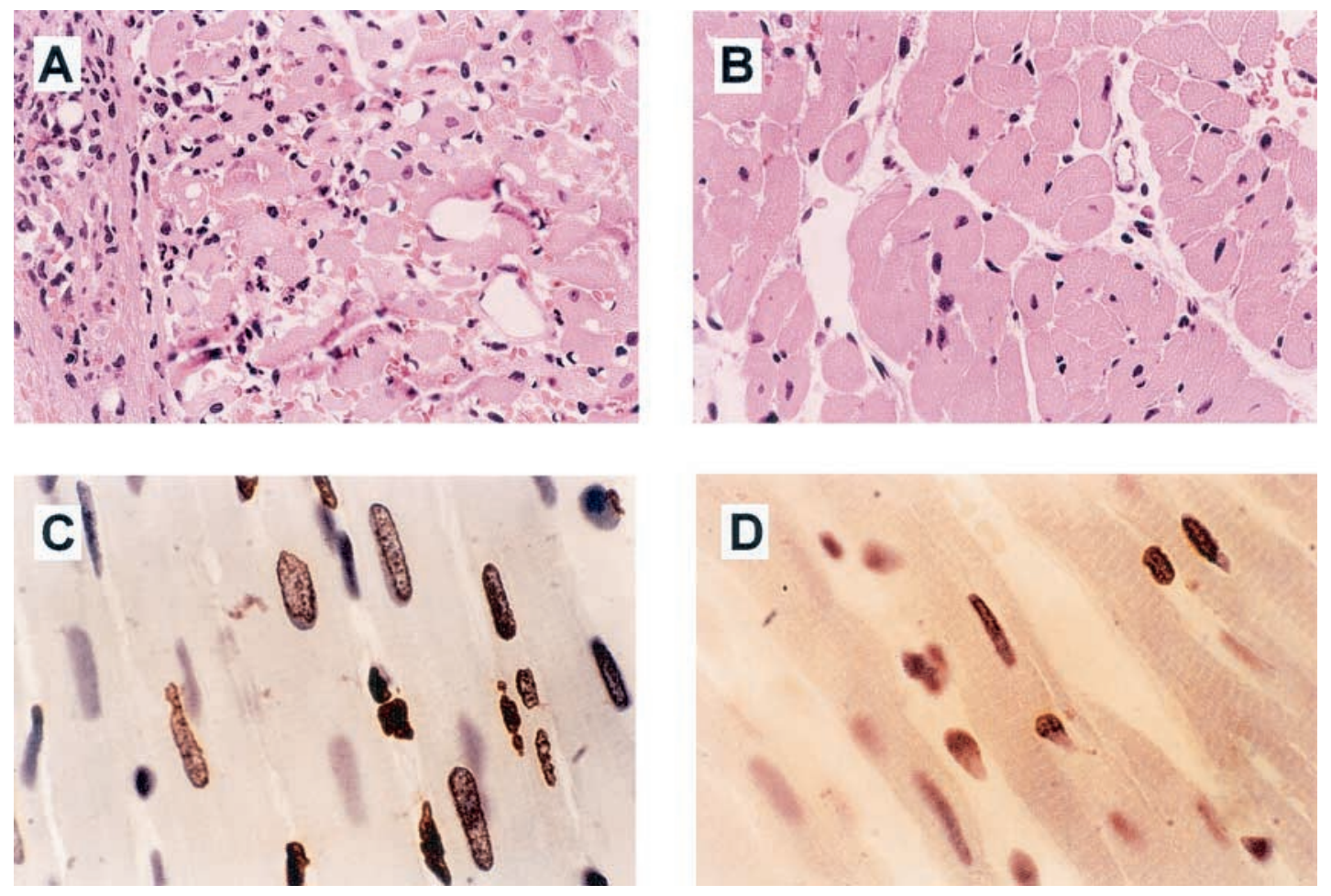

Figure 3. Photomicrographs of ischemic-reperfused (20 minutes of ischemia and 24 hours of reperfusion) myocardium of rats treated either with vehicle (A) or $20,000 \mathrm{U} / \mathrm{kg}$ aprotinin (B). A, Ischemic-reperfused myocardial tissue exhibits numerous leukocytes sequestered inside the coronary vasculature and transmigrated PMNs into the myocardium. Cardiac myocytes are severely injured (ie, necrotic). B, Ischemic-reperfused myocardial tissue after aprotinin treatment exhibits significant reduced PMN adhesion and PMN transmigration. In addition, there were almost no visible signs of cardiac injury. (Original magnification $100 \times$.) C, Photomicrograph of rat heart tissue after 20 minutes of ischemia and 24 hours of reperfusion incubated with terminal deoxynucleotidyltransferase to add residues of digoxigenin nucleotide catalytically to the $3^{\prime}-\mathrm{OH}$ end of DNA and labeled with peroxidase substrate solution. (Original magnification $400 \times$.) Brown reaction product is clearly present in nuclei of apoptotic myocytes in vehicle-treated rats. D, Ischemic-reperfused myocardial tissue of rats treated with aprotinin demonstrated remarkably diminished immunostaining, indicating reduced occurrence of myocardial apoptosis after aprotinin treatment. (Original magnification $400 \times$.)

unlikely because rat hearts have almost no coronary collateral blood flow. In previous studies we have analyzed the area at risk after LAD occlusion. In these experiments we were able to demonstrate, with Evans blue exclusion, $60 \%$ to $70 \%$ area at risk in the left ventricle. However, use of the CK loss method might underestimate the cardioprotective effects.

One important component of the myocardial salvage by aprotinin might be due to its ability to reduce PMN accumulation into the ischemic tissue because aprotinin treatment significantly reduced tissue MPO activity in ischemicreperfused LWFW. Clearly, neutrophils are involved in $\mathrm{MI} / \mathrm{R}$ damage in our model because we observed significant increases in MPO activities in vehicle-treated ischemic myocardial tissue. The effects of aprotinin, however, cannot be attributed to changes in circulating white blood cell counts because bolus injection of aprotinin did not result in a significant decrease in white blood cell counts. These data eliminate the possibility that aprotinin administration in vivo exerted leukopenic effects, a phenomenon known to be cardioprotective in myocardial reperfusion injury. ${ }^{22}$

The precise mechanism of aprotinin-mediated attenuation of PMN accumulation is unclear from the present study. However, there are several possible mechanisms. The extravasation process of circulating PMNs, mediated by endothelial and leukocyte adhesion molecules, starts with PMN rolling along the endothelial surface, followed by firm adhesion, shape change, and transmigration across the vessel wall into the interstitium. ${ }^{23}$ Activated neutrophils produce proinflammatory mediators, including neutral serine proteases (eg, elastase or cathepsin G), ${ }^{24}$ tumor necrosis factor- $\alpha$ (TNF- $\alpha$ ), and oxygen-derived free radicals, which serve to activate additional neutrophils. ${ }^{25}$ Hill and colleagues ${ }^{26}$ could demonstrate that aprotinin inhibits systemic 


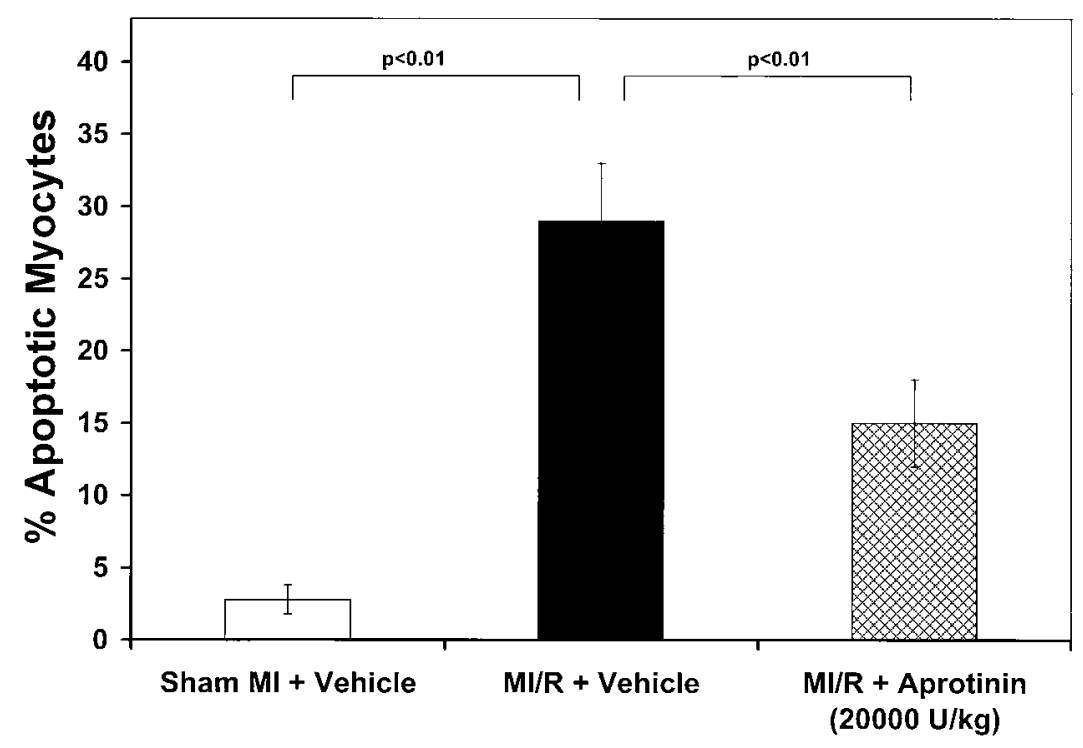

Figure 4. Percentage of apoptotic cardiac myocytes in sham-operated MI rats, MI/R rats treated with vehicle $(\mathrm{n}=$ 5), and MI/R rats treated with $20,000 \mathrm{U} / \mathrm{kg}$ aprotinin $(\mathrm{n}=5)$. Apoptosis was determined with the TUNEL reaction. Values are means \pm SEM.

TNF- $\alpha$ release and subsequent neutrophil CD11b upregulation. This is supported by the observation that TNF- $\alpha-$ stimulated release of platelet-activating factor and leukotriene $\mathrm{B}_{4}$ can be inhibited by serpins and increased by cathepsin $\mathrm{G}$ and human PMN elastase. ${ }^{27}$

In addition to these data, Rice and Weiss ${ }^{28}$ have demonstrated that a serine protease inhibitor was effective in reducing the degradation of fibronectin or elastin in vitro. Therefore administration of an exogenous protease inhibitor results in interruption of the protease-mediated inflammatory cascade and is able to inhibit PMN-endothelial interaction and leukocyte infiltration. Similarly, in an in vitro adhesion assay, aprotinin was able to reduce PMN adhesion to thrombin-activated aortic endothelium (unpublished observation). This property of aprotinin might be one of the key mechanisms by which aprotinin afforded cardioprotection in vivo.

Previous clinical studies have shown that aprotinin demonstrated significant reduction of complement activation during lifesaving ex vivo hemodialysis. ${ }^{29}$ Complement activation plays an important role in myocardial reperfusion injury. Complement activation after MI/R can induce Pselectin and intercellular adhesion molecule expression, and this facilitates neutrophil accumulation. ${ }^{30}$ In this regard administration of aprotinin might be able to partially inhibit complement activation after MI/R, with subsequent reduction of myocardial necrosis and PMN recruitment, because aprotinin was able to inhibit complement-mediated sheep cell hemolysis (data not shown).

Interestingly, complement activation after MI/R induces cardiac myocyte apoptosis. ${ }^{31}$ Gottlieb and colleagues ${ }^{32}$ have demonstrated, in a rabbit model, that MI/R induces apoptosis in reperfused myocardial myocytes and that this might play an important role for delayed myocardial cell death. Apoptosis occurred mainly after reperfusion of the ischemic myocardium and seems to be a contributory mechanism to reperfusion injury. ${ }^{33}$ Our results demonstrating end-labeling of DNA in cardiac myocyte nuclei confirm and extend these findings in a rat model of MI/R. Apoptosis represents a control mechanism in morphogenesis and cell turnover of cells in adult tissues. Apoptosis can be morphologically characterized by cell shrinking, loss of cell contacts, and aggregation of chromatin. Membrane-bound apoptotic bodies are formed that contain intact cell organelles and condensed chromatin. These bodies are phagocytosed by macrophages. Apoptosis can be triggered by cytokines, such as TNF- $\alpha$, which can also contribute to reperfusion injury. ${ }^{34}$ In this regard aprotinin demonstrated beneficial effects on myocardial biochemical function through suppression of TNF- $\alpha .{ }^{35}$ Recently, Buerke and colleagues ${ }^{36}$ were able to demonstrate reduced myocyte apoptosis after insulin-like growth factor I treatment in the same rat model of MI/R. In our study aprotinin reduced the incidence of apoptosis in the ischemic-reperfused myocardium. Because reperfusion-induced apoptosis is independent of neutrophil accumulation in the reperfused myocardium, the antiapoptotic actions of aprotinin in the present experiments are presumably not directly mediated by attenuation of PMN sequestration.

In conclusion, we have demonstrated that administration of aprotinin attenuates both myocardial necrosis and apo- 
ptosis resulting from MI/R. These protective effects could be at least partially attributed to reduced PMN accumulation after aprotinin administration in the reperfused myocardium and subsequent reduced cardiac necrosis. Aprotinin might therefore have important anti-inflammatory effects in addition to its well-known antifibrinolytic effect, which is clinically used for blood loss-lowering actions in cardiac surgery and large general surgical procedures. Our observed cardioprotective effects after aprotinin treatment might represent a strategy for primary prevention of tissue damage mediated by ischemia and reperfusion or similar inflammatory states.

Parts of these studies are included in the thesis of M. Khalil.

\section{References}

1. Forman MB, Kolodgie FD, Jenkins M, Virmani R. Myocardial infarct extension during reperfusion after coronary artery occlusion: pathologic evidence. J Am Coll Cardiol. 1993;21:1245-53.

2. Lefer AM, Tsao PS, Lefer DJ, Ma X-L. Role of endothelial dysfunction in the pathogenesis of reperfusion injury after myocardial ischemia. FASEB J. 1991;5:2029-34.

3. Entman ML, Smith CW. Postreperfusion inflammation: a model for reaction to injury in cardiovascular disease. Cardiovasc Res. 1994;28: 1301-11.

4. Weiss SJ. Tissue destruction by neutrophils. N Engl J Med. 1989;320: 365-76.

5. Caughey GH. Serine proteinases of mast cell and leukocyte granules-a league of their own. Am J Respir Crit Care Med. 1994;150: S138-42.

6. Mulligan MS, Desrochers PE, Chinnaiyan AM, Gibbs DF, Varani J, Johnson $J$, et al. In vivo suppression of immune complex-induced alveolitis by secretory leukoproteinase inhibitor and tissue inhibitor of metalloproteinases. Proc Natl Acad Sci U S A. 1993;90:11523-7.

7. Oeveren van W, Jansen NJ, Bidstrup BP, Royston D, Westaby S, Neuhof H, et al. Effects of aprotinin on haemostatic mechanism during cardiopulmonary bypass. Ann Thorac Surg. 1987;44:640-5.

8. Lord RA, Roath OS, Thompson JF, Chant AD, Francis JL. Effect of aprotinin on neutrophil function after major vascular surgery. $\mathrm{Br} \mathrm{J}$ Surg. 1992;79:517-21.

9. Lefer AM, Spath JA. Preservation of myocardial integrity by a protease inhibitor during acute myocardial ischemia. Arch Int Pharmacodyn Ther. 1974;211:225-36.

10. Wendel HP, Heller W, Michel J, Mayer G, Ochsenfahrt C, Graeter U, et al. Lower cardiac troponin $\mathrm{T}$ levels in patients undergoing cardiopulmonary bypass and receiving high-dose aprotinin therapy indicate reduction of perioperative myocardial damage. J Thorac Cardiovasc Surg. 1995;109:1164-72.

11. Zimmerman BJ, Granger DN. Reperfusion-induced leukocyte infiltration: role of elastase. Am J Physiol 1990;259:H390-4.

12. Inauen W, Granger DN, Meininger CJ, Schelling ME, Granger HJ, et al. Inhibition of neutrophil superoxide generation by $\alpha 1$-antichymotrypsin. J Immunol. 1991;146:2388-93.

13. Buerke M, Weyrich AS, Lefer AM. Isolated cardiac myocytes are sensitized by hypoxia-reoxygenation to neutrophil related mediators. Am J Physiol. 1994;266:H128-36.

14. Kjiekhus JK, Sobel BE. Depressed myocardial creatine phosphokinase activity following experimental myocardial infarction in rabbit. Circ Res. 1970;27:403-14
15. Lefer AM, Tsao P, Aoki N, Palladino MA Jr. Mediation of cardioprotection by transforming growth factor-beta. Science. 1990;249: 61-4.

16. Rosalki SB. An improved procedure for serum creatine phospokinase activity following experimental myocardial infarction in rabbit. Circ Res. 1970;27:403-14.

17. Gornall AG, Bardowill CT, David MM. Determination of serum protein by means of the biuret method. J Biol Chem. 1949;177:751-66.

18. Bradley PP, Priebat DA, Christensen RD, Rothstein GR. Measurement of cutanous inflammation: estimation of neutrophil content with an enzyme marker. J Invest Dermatol. 1982;78:206-9.

19. Mullane KM, Kraemer R, Smith B. Myeloperoxidase activity as a quantitative assessment of neutrophil infiltration into ischemic myocardium. J Pharmacol Methods. 1985;14:157-67.

20. Buerke M, Murohara T, Lever AM. Cardioprotective effects of as C1 esterase inhibitor in myocardial ischemia and reperfusion. Circulation. 1995;91:393-402.

21. Engler RL. Free radical and granulocyte-mediated injury during myocardial ischemia and reperfusion. Am J Cardiol. 1989;63:19E-23E.

22. Gottlieb RA, Engler RL. Apoptosis in myocardial ischemia-reperfusion. Ann N Y Acad Sci. 1999;874:412-26.

23. Butcher EC. Leukocyte-endothelial cell recognition: three (or more) steps to specify and diversity. Cell. 1991;67:1033-6.

24. Rubin H. The biology and biochemistry ofantichymotrypsin and its potential role as a therapeutic agent. Biol Chem. 1992;373:497-502.

25. Sands H, Hook JB. Pharmacology and pharmacokinetics of LEX-032, a bioengineered serpin. The first of a potential new class of drugs. Drug Metab Rev. 1997;29:309-28.

26. Hill GE, Alonso A, Spurzem JR, Stammers AH, Robbins RA. Aprotinin and methylprednisolone equally blunt cardiopulmonary bypassinduced inflammation in humans. J Thorac Cardiovasc Surg. 1995; 110:1658-62.

27. Camussi G, Tetta C, Bussolino F, Baglioni C. Synthesis and release of platelet activating factor is inhibited by plasma $\alpha 1$-antichymotrypsin and is stimulated by proteases. J Exp Med. 1988;68:1293-306.

28. Rice WG, Weiss SJ. Regulation of proteolysis at the neutrophilsubstrate interface by secretory leukoprotease inhibitor. Science. 1990; 249:178-81

29. Himmelfarb J, Holbrook D, McMonagle E. Effects of aprotinin on complement and granulocyte activation during ex vivo hemodialysis. Am J Kidney Dis. 1994;24:901-6.

30. Buerke M, Prufer D, Dahm M, Oelert H, Meyer J, Darius H. Blocking of classical complement pathway inhibits endothelial adhesion molecule expression and preserves ischemic myocardium from reperfusion injury. J Pharmacol Exp Ther. 1998;286:429-38.

31. Vakeva AP, Agah A, Rollins SA, Matis LA, Li L, Stahl GL. Myocardial infarction and apoptosis after myocardial ischemia and reperfusion: role of the terminal complement components and inhibition by anti-C5 therapy. Circulation. 1998;97:2259-67.

32. Gottlieb RA, Burleson KO, Kloner RA, Babior BM, Engler RL. Reperfusion injury induces apoptosis in rabbit cardiomyocytes. J Clin Invest. 1994;94:1621-8.

33. Fliss H, Gattinger D. Apoptosis in ischemic and reperfused rat myocardium. Circ Res. 1996;79:949-56.

34. Raff MC. Social controls on cell survival and cell death. Nature. 1992;356:397-400.

35. Bull DA, Connors RC, Albanil A, Reid BB, Neumayer LA, Nelson R, et al. Aprotinin preserves myocardial biochemical function during cold storage through suppression of tumor necrosis factor. $J$ Thorac Cardiovasc Surg. 2000;119:242-50.

36. Buerke M, Murohara T, Skurk C, Nuss C, Tomaselli K, Lefer AM. Cardioprotective effect of insulin-like growth factor I in myocardial ischemia followed by reperfusion. Proc Natl Acad Sci U S A. 1995; 92:8031-5. 